DOI: $10.14746 /$ por.2019.1.4

\title{
DIE UMGESTALTUNG DES KOLLEKTIVEN GEDÄCHNISSES DIE IDEOLOGISCHE FUNKTION DES UKRAINISCHEN INSTITUTS FÜR NATIONALES GEDÄCHNIS IN 2014-2017
}

\author{
Mikhail MinaKov ${ }^{1}$ \\ (Europa-Universität Viadrina Frankfurt Oder)
}

Schlüsselwörter: Ideologie, ideologisches Monopol, kollektive Erinnerung, Ukraine, Euromaidan, das Ukrainische Institut für Nationales Gedächtnis, postsowjetische Politik

Keywords: ideology, ideological monopoly, collective memory, Ukraine,

Euromaidan, Ukrainian Institute of National Memory, post-Soviet politics

\begin{abstract}
Mikhail Minakov, DIE UMGESTALTUNG DES KOLLEKTIVEN GEDÄCHNISSES. DIE IDEOLOGISCHE FUNKTION DES UKRAINISCHEN INSTITUTS FÜR NATIONALES GEDÄCHNIS IN 2014-2017. „PORÓWNANIA” 1 (24), 2019. T. XXIV, S. 31-43. ISSN 1733-165X. Nach dem Euromaidan entwickelte sich die Ukraine widersprüchlich. Neben der Demokratisierung und Europäisierung verbreiteten sich auch konservative Ideologien und Einschränkungen der bürgerlichen Freiheiten. Diese letzteren Tendenzen waren mit dem Versuch verbunden, ein ideologisches Monopol (ein System der ideologischen Kontrolle, das in der UdSSR existierte und in den Dekommunisierungsprozessen von 1989-91) im Interesse der heutigen herrschenden Gruppen zu etablieren. Eine der Institutionen, die die Möglichkeit der Wiederbelebung des ideologischen Monopols ergänzen, ist das Ukrainische Institut für Nationales Gedächtnis. In diesem Text argumentiert der Autor, dass sich das Institut nach mehreren Reformen zwischen 2006 und 2018 zu einer staatlichen Einrichtung entwickelt hat, die konservative Kulturpolitik und eine Politik der illiberalen Identität betreibt, die die staatsbürgerliche demokratische Konsolidierung der Ukraine untergräbt. Anhand der Analyse der politischen Rolle und der bisherigen Erfolge des Instituts gelangt der Autor zu dem Schluss, dass die derzeitige Kulturpolitik und die Organisationspraktiken des Instituts ein neues postsowjetisches ideologisches Instrumentarium geschaffen haben, mit dem Machteliten effektiv ein konservatives ideologisches Monopol in der Ukraine aufbauen können.
\end{abstract}

1 E-mail: mikhailminakov1971@gmail.com 
Abstract: Mikhail Minakov, TRANSFORMATION OF THE COLLECTIVE MEMORY. THE IDEOLOGICAL FUNCTION OF THE UKRAINIAN INSTITUTE OF NATIONAL MEMORY IN 2014-2017. "PORÓWNANIA" 1 (24), 2019. Vol. XXIV, P. 31-43. ISSN 1733-165X. The post-Euromaidan's Ukraine has developed in a contradictory way: together with democratization and Europeanization, there was also a spread of conservative ideologies and limitation of civic freedoms. The latter tendencies were connected with the attempts at establishing ideological monopoly - a system of ideological control that existed in USSR and disappeared in the decommunization processes of 1989-91 - in the interests of nowadays' ruling groups. One of those institutions adding to the possibility of revival of the ideological monopoly is the Ukrainian Institute of National Memory. In this paper, the author argues that after several reforms between 2006 and 2018, the Institute evolved into a state body providing conservative cultural policies and illiberal identity policies undermining Ukraine's civic democratic consolidation. Based on the analysis of the Institute's political role and achievements so far, the author concludes that the current cultural policies and organizational practices of the Institute (and similar organizations) have created a new post-Soviet ideological toolkit enabling power elites to effectively establish conservative ideological monopoly in Ukraine.

Am 24. Mai 2018 gab eine Gruppe ukrainischer Marinesoldaten eine Demarche gegen den Präsidenten Poroschenko während seines Besuches bei der Marinebrigade ab. Der Präsident war gekommen, um eine neue Uniform und neue Formen des militärischen Grußes zu präsentieren. Aber die Soldaten wollten diese Neueinführungen nicht akzeptieren, weil für sie ihre eigenen alten schwarzen Barette und der Gruß eine Frage der Ehre waren. In der Folge wurden die ungehorsamen Marinesoldaten noch am selben Tag aus dem Armeedienst entlassen.

Für Marinesoldaten sind die schwarzen Barette ein Symbol der neuen ukrainischen Armee. Seit 1992, als die sowjetische Armee auf der Krim in zwei Teile - einen ukrainischen und einen russischen - gegliedert wurde, hatten die Marinesoldaten auf schwarzen Baretten ihr Gelöbnis abgelegt, der Ukraine treu zu dienen. In diesem Zusammenhang hatte die neue Armee auch eigene Militärrituale formiert, zum Beispiel den Gruß „Diene der Ukraine!“

Nach dem Sieg des „Euromaidan“ von 2014 führte die neue Regierung viele symbolische und ideologische Innovationen ein. Einige davon haben viel mit der kollektiven Erinnerung zu tun. Alle Ereignisse und Symbole aus der Zeit vor 2014 mussten umgewertet werden. Auch im Bereich des Militärs wurden nicht nur jene Sachverhalte ausgewechselt, die an die Sowjetunion geknüpft waren, sondern auch jene, die der neuen Staatsideologie seit 2014 nicht entsprechen. Beispielsweise sollen Soldaten nun eine neue Uniform tragen und den Gruß benutzen, der mit der nationalistischen ukrainischen Bewegung assoziiert wird: "Ruhm der Ukraine!" Ein tiefgreifender Konflikt war entstanden. Einerseits darf ein Präsident, der auch Oberbefehlshaber ist, keine Befehlsverweigerung in der Armee unbestraft lassen. Andererseits sind die "Schwarzen Barette“ offensichtlich gegenüber der nachsowjetischen Ordnung loyal: sie blieben ihrem nachsowjetischen Militäreid und ihrer Regimentstradition treu. 
Wir sehen also einen Streitfall von zwei Erinnerungspolitiken. Die ungehorsamen Soldaten blieben der Tradition treu, die mit der ukrainischen Unabhängigkeit begründet worden war.

Nachdem im Jahr 2015 die neuen Machtgruppen des Postmaidan eine neue Erinnerungspolitik in Form der sogenannten "Dekommunisierungsgesetze“ formuliert hatten, wurden auch viele Staatsregeln und Militärtraditionen modifiziert. Unter anderem versuchten diese Gruppen die neuen Grußformeln und Symbole einzuführen, die mit der Ukrainischen Volksrepublik (1918-21) und/oder Organisationen der ukrainischen Nationalisten assoziiert werden. Gleichzeitig wurde das Erbe der sowjetischen (1917-1991) und postsowjetischen (1991-2014) Ukraine entweder verdammt oder vergessen. Jetzt sind die Erinnerungen an die sowjetischen und vormaidanischen Perioden Gegenstand staatlicher Umordnung, Modifikation und Kontrolle. Allerdings ist die neue Politik der Erinnerungskontrolle umstritten.

In meinem Referat möchte ich über diese Erinnerungskontrolle sprechen, indem ich hier die Arbeit des Ukrainischen Institutes für Nationales Gedächtnis in Bezug auf die ideologische Funktion des postsowjetischen Staates analysiere.

Die Hauptthese lautet: Die Leitaufgabe des heutigen Ukrainischen Institutes für Nationales Gedächtnis ist die Herstellung eines ideologischen Monopols, bei dem die Erinnerungskontrolle durch die regierenden Machtgruppen eine Kernfrage darstellt.

Um diese These zu unterfüttern, gehe ich in meinem Beitrag auf folgende Fragen ein: erstens auf die ideologische Rolle der kollektiven Erinnerung, zweitens auf die ideologische Funktion des postsowjetischen Staates und drittens auf die Aktivitäten des Instituts zwischen 2015 und 2018.

\section{Die ideologische Rolle der kollektiven Erinnerung}

Erinnerung ist ein kompliziertes kognitives Geschehen, das sich auf nicht mehr existierende Situationen bezieht. Jede Art der Erkenntnis ist höchst kompliziert. Doch die Erinnerung ist noch komplizierter, denn ihren Gegenstand gibt es nicht mehr. Darum gilt das Gedächtnis des Menschen als uralter Gegenstand für philosophische, logische, biologische und medizinische Untersuchungen.

Die Erinnerung besteht aus drei Elementen: einem sich erinnernden Subjekt, dem Vorgang des Erinnerns und dem Objekt der Erinnerung (z.B. ein Ereignis, auf das sich die Erinnerung bezieht). In der Erinnerung aktualisiert das Subjekt das Vergangene und macht es in der Gegenwart präsent (Berek, 2009; Olik, 2007).

Das sich erinnernde Subjekt ist immer ein einzelner Mensch. Doch Wissenschaftler, die sich mit der kollektiven Erinnerung beschäftigen, machen mitunter den Fehler, die Fähigkeit des Erinnerns allzu direkt auf ein Kollektiv zu übertragen. Das zeigt sich am Beispiel einer jüngst veröffentlichen Studie zu kollektiven Erinne- 
rungen der Europäer. Die Autoren behandeln nicht nur die individuellen Subjekte der Erinnerung, sondern auch „kollektive, korporative Akteure wie z. B. Museen, politische Parteien oder die Regierungen von Staaten“ (Gerhards et al., 2017: 18). Hunderte wissenschaftliche Bücher in Geschichte, Soziologie und Politologie fußen auf der Annahme, kollektive Erinnerungen seien aus individuellen Erinnerungen herleitbar.

Ohne Zweifel hat die Metapher des kollektiven Gedächtnisses sehr solide Heuristik, wie uns Paul Ricœur, Jan und Aleida Assmann, Maurice Halbwachs oder Pierre Nora gezeigt haben (Ricœur, 1986; Assmann, 2006; Halbwachs, 1985; Nora, 2005). Sie hilft uns, Kultur, Gesellschaft, Gemeinschaft oder Institutionen besser zu verstehen. Das Gebiet der sogenannten "kollektiven Erinnerungen” ist so reich, dass Jan und Aleida Assmann (2006) zwischen drei verschiedenen Formen des Gedächtnisses unterscheiden mussten: dem kulturellen, dem sozialen und dem kollektiven Gedächtnis.

Gleichzeitig ist es wichtig, folgendes zu unterstreichen: Alle nichtindividuellen Gedächtnisformen sind nicht nur an vergangene menschliche Erfahrungen geknüpft, sondern auch an heutige imaginierte Bilder der Vergangenheit. Die soziale Einbildung benutzt diese Bilder des Vergangenen, um die Grenzen (z.B. zwischen "uns" und „Fremden”) zu schaffen oder um die Bevölkerung eines Staates in eine Nation zu binden. Diesen Aspekt der kollektiven Erinnerung zeigten uns Benedict Anderson (1991) und seine Nachfolger (z.B. Acquisti \& Gross, 2007) mit der Metapher der „imagined communities".

Ich möchte hier betonen, dass die ideologische Funktion der kollektiven Erinnerung aus der Struktur der Erfahrung selbst kommt. Wie die soziale Phänomenologie gezeigt hat, ist die Erfahrung gleichzeitig individuell und sozial bzw. intersubjektiv (Schütz \& Luckmann, 2003; Berger \& Luckmann, 1967). Die intersubjektive Struktur der Erfahrung ist tiefer als die Subjekt-Objekt-Dichotomie und bringt individuelle Erfahrung mit den vier Welten zusammen: Umwelt, Mitwelt, Vorwelt und Nachwelt.

Die Umwelt bedeutet eine Welt, in der ein Mensch in unmittelbarer Präsenz mit einem Anderen ist; hier kann man reziproke/gegenseitige direkte Beziehungen eingehen und an sozialen Handlungen teilnehmen. In der Umwelt ist der Sinn immer auf individuelle Erfahrungen bezogen.

Die Mitwelt umfasst alle Menschen, die für die individuellen Akteure prinzipiell erreichbar sind: sie leben zu derselben Zeit auf der Erde, sind aber geografisch voneinander distanziert. In dieser haben wir keine unmittelbare Erfahrung mit entfernten Zeitgenossen. Man braucht bestimmte Medien, um etwas über die Bewohner der Mitwelt zu wissen. Darum muss sich das Bild der Anderen hier an typisierten Erwartungen orientieren, die in der Regel starken sozialen Normierungen unterworfen sind.

Es ist für unseren Themenbezug auch sehr wichtig, dass die soziale Vorwelt für die Menschen weder unmittelbar noch mittelbar erreichbar ist. Alle Bedeutungen 
dieser Welt kommen aus der Vergangenheit: sie wurden einmal begriffen und sind uns durch zeitspezifische Medien in verschiedenen Formen überliefert. Darum ist hier die Möglichkeit des intersubjektiven Verstehens stark modifiziert.

Was weiterhin für unser Thema eine wichtige Rolle spielt, ist die Tatsache, dass das individuelle Gedächtnis immer nur ein kleiner Teil der Erfahrung ist. Die meisten Bedeutungen sind anonymer Natur und kommen - mit typisierenden Medien der Sprache, Institutionen usw. - aus Mitwelt, Vorwelt und Nachwelt (Schütz \& Luckmann, 2003; Berger \& Luckmann, 1967). Die Erinnerungen, die stark mit der Vorwelt und der Mitwelt verknüpft sind, haben wenig mit vergangenem Geschehen zu tun.

Diese Schlussfolgerung bedeutet natürlich nicht, dass die Erinnerung nicht wichtig ist. Sie ist von Bedeutung, doch in einem spezifischen Sinne. Alle Arten des kollektiven Gedächtnisses dienen ideologischen Zielen: Menschen zu sammeln, zu teilen, zu mobilisieren und ihrem Leben einen ideologischen Inhalt zu geben. Hier beziehe ich mich auf Ideologietheorien von Clifford Geertz und Paul Ricœur, die eine prinzipielle Zweideutigkeit der Ideologie gezeigt haben: Einerseits organisiert die Ideologie sinnvoll die menschlichen Kollektive, Kulturen usw., andererseits aber teilt sie die Menschen in Gruppen, symbolisiert und schreibt ihre Unterschiede fest. Damit stellen Ideologien auch eine Herausforderung für das gegenseitige Verständnis von Menschen dar (Geertz, 1964; Ricœur, 1986).

Kollektive Erinnerungen sind, so können wir schlussfolgern, immer ideologisch formiert. Das bedeutet, dass das sogenannte nationale Gedächtnis und die kollektiven Erinnerungen (zusammen mit anderen ideologischen Formen) den Zugang $\mathrm{zu}$ den nationalen Ressourcen und staatlichen Machtzentren mitbestimmen. Das nationale Gedächtnis und die kollektiven Erinnerungen formieren die kollektiven Identitäten, und bestimmen, welche sozialen Gruppen zur politischen Gesellschaft gehören und welche Gruppen ausgeschlossen sind. Wenn politische Systeme eine Institution gründen und damit die ideologisch typisierende Gewalt des Gedächtnisses den spezifischen Machtgruppeinteressen unterordnen, dann können wir die Gefahr eines neuen illiberalen, autoritären oder sogar totalitären Staates erkennen.

\section{Ideologische Funktion des Staates}

Die heutigen Politikwissenschaftler sind zu dem Konsens gekommen, dass es vier fundamentale Eigenschaften des modernen Staates gibt. Erstens: Das Monopol der Staatsregierung über die legitimen Mittel der Macht, die die Kontrolle über die Bevölkerung auf einem bestimmten Territorium ermöglichen. Zweitens: Dasselbe Monopol ermöglicht auch die Verteidigung der Macht und der kontrollierten Bevölkerung vor einer externen Bedrohung. Drittens: Die Staatsmacht muss in der Lage sein, der kontrollierten Bevölkerung die notwendigen administrativen und 
rechtlichen Dienstleistungen zu gewähren. Und viertens: Die Regierung hat das Monopol auf die Besteuerung der Bevölkerung (Tilly 1992: 23 u.a.; Ghani \& Lockhart 2008: 128 u.a.).

Die ideologischen Dienstleistungen stellen mithin keine notwendige Staatsfunktion dar. Doch in der Regel wird eine solche von modernen Staaten praktiziert. Unter den Regierungsbehörden, die diese unsichtbare Funktion erfüllen, sind Organisationen wie Bildungs- und Kultusministerien, staatliche Ausschüsse für Medien, Film u.a. Eine ganze Reihe von Staatsbehörden und eine bestimmte Menge nationaler Ressourcen stehen hierbei auch dem Ideologie- und Identitätsmanagement zur Verfügung.

In extremen Fällen ermöglicht diese ideologische Dienstleistung totalitäre Ideokratien wie in Nazi-Deutschland, der stalinistischen Sowjetunion oder im nachrevolutionären Iran. In diesen Fällen ist die dominierende Ideologie immer gut artikuliert und ihr Monopol durch spezifische Institutionen garantiert.

Aber auch liberale Staaten üben ideologische Funktionen aus. Hier besteht allerdings zumeist ein ideologischer Pluralismus, der von verschiedenen demokratischen Institutionen abgesichert ist. In diesen Gesellschaften gibt es vielfältige kollektive Akteure, die an der ideologischen Produktion teilnehmen. In den USA könnten z.B. das Weiße Haus und der Capitol Hill (also die Regierung) ebenso genannt werden wie die Massenkultur von Hollywood, die Wall Street als finanzielles Machtzentrum, die Madison Avenue mit ihren Werbeagenturen und die M-Street in Washington, in der viele Think Tanks angesiedelt sind. Zusammen produzieren alle genannten Instanzen spezifische Ideologiekomplexe des späten Konsumkapitalismus. Da aber diese ideologischen Machtzentren diversifiziert und antagonistisch bleiben, gibt es hier geringe Chancen für ein ideologisches Monopol.

Die posttotalitären Staaten mit ihren traumatischen historischen Erfahrungen versuchen in der Regel, die Rückkehr eines ideologischen Monopols zu verhindern. Viele postsowjetische Länder haben klare Verfassungsbestimmungen, die das ideologische Monopol und die staatliche Zensur direkt verbieten. Artikel 15 der Verfassung der Ukraine lautet:

„Das öffentliche Leben in der Ukraine basiert auf den Prinzipien der politischen, wirtschaftlichen und ideologischen Vielfalt. Keine Ideologie kann vom Staat als verbindlich anerkannt werden. Die Zensur ist verboten [... $]^{\prime \prime}$ (Verfassung). ${ }^{2}$

In diesem Kontext spielen die Lustrationsinstitute der zentraleuropäischen Staaten, die dem „Therapieren“ des Gedächtnisses dienen sollten, eine bemerkenswerte Rolle. Die Tschechoslowakei gründete das Institut für Nationales Gedächtnis im Jahre 1991; Ungarn folgte im Jahr 1993 und Litauen im Jahr 2000 (Portnow, 2014). In jenen Jahren dienten die Institute für nationales Gedächtnis als Machtzentren, die die kommunistische Vergangenheit zu bewältigen versuchten. Sie sollten, auch wenn

2 Hier und des Weiteren werden alle Zitate aus den Gesetzen vom Autor übersetzt. 
dies nicht direkt ausgesprochen wurde, das kollektive Gedächtnis im Hinblick auf kommunistische Regimes formen. Sie waren beauftragt, für eine freie kollektive $\mathrm{Zu}$ kunft zu sorgen und jene Personen, die sich mit kriminellen Aktivitäten der ehemaligen kommunistischen Regierungen befleckt hatten, aus dem öffentlichen Leben zu drängen. Obwohl noch nicht abzusehen ist, wie effizient und therapeutisch diese Institute waren, dienten sie zweifellos der Sache der posttotalitären Emanzipation.

Später, schon im 21. Jahrhundert, waren auch die ukrainischen Eliten imstande, das Institut für Nationales Gedächtnis zu gründen. Die Gründung erfolgte zu einer Zeit, als totalitäre sowjetische Strukturen bereits nicht mehr existierten und die Ukraine sich zu einem ideologisch, politisch und kulturell pluralistischen Land entwickelt hatte. In diesem Zusammenhang hatte das Institut gegensätzliche Ziele zu erfüllen: Einerseits signalisierte es einen ideologischen Gestaltungsanspruch der Regierung, andererseits war nicht geklärt, wie das Verhältnis zwischen dem sowjetischen und dem zaristischen Erbe zu behandeln sei.

\section{Das Ukrainische Institut für nationales Gedächtnis als ideologisches Instrument}

Das Ukrainische Institut für Nationales Gedächtnis wurde von Präsident Juschtschenko inspiriert und im Jahr 2006 von der ukrainischen Regierung gegründet (Кабінет Міністрів України, 2006). Damals legte die Regierung als zentrale Exekutivbehörde mit einem Dekret für diese Institution die folgenden Ziele fest:

- „Die Lenkung der Aufmerksamkeit der Gesellschaft auf ihre eigene Geschichte und die Verbreitung objektiver Informationen darüber in der Ukraine und der Welt"

- „Die Wiederherstellung und Erhaltung des nationalen Gedächtnisses des ukrainischen Volkes"

- „Das Studium der Stadien des Kampfes für die Wiederherstellung der ukrainischen Staatlichkeit im 20. Jahrhundert, der historischen Vergangenheit des ukrainischen Volkes einschließlich aller Formen von Repression“

- „Die Erinnerung an die Opfer des Holodomor und politischer Repression und auch an die Teilnehmer des nationalen Befreiungskampfes" (Ibid., 1-2)

Präsident Juschtschenko und die Gruppen der Intellektuellen und Politiker, die ihn unterstützten, hatten mithin die Hoffnung, dass sich die Behörde mit der "objektiven ukrainischen Geschichte" und der wahren ukrainischen kollektiven Identität systematisch beschäftigen würde. Georgii Kasyanov, der diese Hoffnungen analysiert hat, schrieb, dass diese Gruppe ein neues gigantisches „Ministerium der Wahrheit" erwartete (Kasyanov, 2018: 136). Es ist wichtig darauf hinzuweisen, dass die Gründung des Instituts ohne gesellschaftlichen oder elitären Konsens geschah, sondern auf Grundlage der politischen Konjunktur (ebd.). Von Anfang an nutzte 
die regierende Gruppe der „Orangenen Revolution“ die Zeit ihrer Dominanz, um ihre konservative und latent nationalistische Ideologie in der Ukraine zur Hegemonie zu verhelfen.

Während der Juschtschenko-Periode arbeitete das Institut vor allem an zwei Projekten: an der Vertiefung der Erinnerung an den Holodomor und an der Etablierung der Führer der "Ukrainischen Aufständischen Armee“ als Helden. Beide Projekte umfassten geschichtliche und ideologische Elemente. Wissenschaftler des Instituts untersuchten Archivdokumente, um die Ursachen und Nachfolgen des Holodomor besser zu verstehen. Gleichzeitig war das Institut verantwortlich für öffentliche Aktivitäten, um den Holodomor nicht nur als Tragödie der ukrainischen Bevölkerung, sondern auch als Genozid am ukrainischen Volk zu brandmarken. Das Institut bereitete auch ein Buch über die Holodomor-Opfer vor. Dieses Buch sollte die These von Präsident Juschtschenko beweisen, dass zirka zehn Millionen Ukrainer als Opfer des organisierten Hungers gestorben waren (Juschtschenko, 2009). Diese Zahl wurde auch von anderen Politikern in den Raum gestellt; die Wissenschaft hatte es $\mathrm{zu}$ beweisen.

Ähnlich gelagert war der Fall von Stepan Bandera, des Anführers der „Organisation Ukrainischer Nationalisten“: der Politiker Juschtschenko ehrte Bandera als "Helden der Ukraine" und das Personal des Instituts musste die Entscheidung belegen und rechtfertigen.

2010 wurde das Institut erneut zum Opfer der politischen Konjunktur. Als einzige staatliche Behörde wurde sie von der Regierung des neuen Präsidenten Janukowitsch liquidiert und als Dezernat im Ministerkabinett wiedereröffnet (Кабінет Міністрів України, 2011). Diesmal wurde es als Forschungseinrichtung unter der Leitung des alten Kommunisten Walerii Soldatenko etabliert. Zwischen 2010 und 2014 war das Institut eine „Low-Profile-Institution“; während dieser Zeit konnten die Wissenschaftler ohne große politische Aufsicht arbeiten. Damals spielte das Institut fast keine politische und ideologische Rolle in der Ukraine.

Den Höhepunkt seines Einflusses erreichte das Institut nach dem „Euromaidan" im Jahre 2014. Im Juli wurde das Institut erneut durch eine Kabinettsverordnung geschlossen und als eigene Staatsbehörde wiedereröffnet (Кабінет Міністрів України, 2014a). Ehrgeizig wurden folgende Hauptziele formuliert:

- Die Erforschung der ukrainischen Staatsbildung und der Verbreitung der Ergebnisse in der Ukraine und der Welt;

- „das Gedenken an die Mitglieder der ukrainischen Befreiungsbewegung und -kriege, der Opfer des Holodomor und der politischen Repressionen, an die Kämpfer für die Unabhängigkeit, Souveränität und territoriale Integrität der Ukraine sowie an die Teilnehmenden an Anti-Terror-Operationen“;

- „die Wiederherstellung des nationalen Gedächtnisses des ukrainischen Volkes und die Verhinderung der Verwendung von Symbolen totalitärer Regime"; 
- „die Erziehung zu Patriotismus, nationalem Bewusstsein und zur aktiven Beteiligung der ukrainischen Bürger“;

- Die Erforschung des historischen Erbes (Кабінет Міністрів України, 2014b).

Diese Gesetzbestimmungen haben das Institut und seinen neuen Leiter - den nationalkonservativen Ideologen Wolodymyr Vyatrowytsch - zu einflussreichen Akteuren der ukrainischen Politik gemacht. Dieser Einfluss war bereits vorher durch die verabschiedeten „Dekommunisierungsgesetze“ abgesichert gewesen. Diese bestehen aus vier Gesetzen, die vom ukrainischen Parlament ohne Debatten und trotz Einsprüchen seitens der Parlamentsjuristen angenommen wurden (Kasyanov, 2018: 146). Unter den vielen Normen, die durch diese Gesetze eingeführt wurden $^{3}$, gab es u.a. folgende ideologische Satzungen:

- Die Gleichsetzung des Kommunismus und Nationalsozialismus als totalitäre "Regimes" und das Verbot ihrer Symbole;

- Die Vergabe eines besonderen rechtlichen Status an die Kämpfer für die Unabhängigkeit der Ukraine im 20. Jahrhundert;

- Die Umbenennung von Städten und Straßen;

- Die Einführung neuer Feiertage.

Diese drei Normen sowie die weiteren Änderungen bei Straf- und Verwaltungsvorschriften sowie spezifische Anwendungspraktiken gaben dem Institut Gelegenheit, ein ideologisches Monopol in der Ukraine zu etablieren.

Die Funktion der symbolischen Kontrolle des Instituts ist ähnlich zu beurteilen wie die Praktiken des Komsomol zu Zeiten der Sowjetunion. Während der Feiertage oder auch in sozialen Netzwerken beobachten das Institut, die Polizei und die Netzwerke assoziierter Aktivisten, welche Symbole und Texte von Ukrainern benutzt werden. So wird die Verwendung des St.-Georgs-Bändchens während der jährlichen Feierlichkeiten zum Ende des Zweiten Weltkriegs bestraft (Zik.ua, 2018). Ein anderes Beispiel betrifft einen Studenten der Nationalen Iwan-Franko-Universität in Lwiw, der dafür bestraft wurde, Abbildungen von Bücherfotografien von Lenin und Marx im Netz gepostet zu haben (Hromadske - Radio, 2017).

Gleichzeitig rufen die Symbole, die mit dem Nationalsozialismus assoziiert werden, keine oder nur schwache Reaktionen seitens der Regierung und des Instituts hervor. Zum Beispiel wurde im April 2018 in Lwiw das Fest der SS-Abteilung "Galizien" gefeiert, bei dem die Anwendung und das Tragen von Nazi-Symbolen nicht zur Bestrafung der Täter führten (Hromadske -Radio, 2018). Im Gebäude der Regionalverwaltung fand eine Ausstellung von Kinderzeichnungen statt, die der SS-Abteilung "Galizien” gewidmet waren (Zaxid.net, 2018).

Noch stärkere Bemühungen unternimmt das Institut bei der „Dekommunisierung" von Städten und Straßen. So wurden mehr als 1.500 Städte und Dörfer umbenannt oder haben ihren alten vorsowjetischen Namen bekommen (УIHП,

3 Auf Englisch sind diese Gesetze hier nachzulesen: [http:/ /www.memory.gov.ua/laws?page=2]. 
2018a). Hinter diesen Umbenennungen verbirgt sich eine ideologische Metaphysik, vergleichbar mit der sowjetischen Praxis der Benennung von Städten und Straßen. So zum Beispiel die Stadt Artemiwsk, deren Sünde es war, in Verbindung mit Artem, dem Gründer der sowjetischen Ukraine, zu stehen. Daraufhin bekam die Stadt ihren vorsowjetischen Namen „Bachmut” zurück, da dieser weder imperialistisch noch sowjetisch konnotiert war. Die Stadt Kirovograd, die mit Sergei Kirow, dem Opfer der stalinistischen Verschwörung, assoziiert wurde, wurde gegen den Willen der Stadtbevölkerung in „Kropywnytskyj” umbenannt. Der vorsowjetische Name der Stadt - "Jelisawetgrad" - habe den Ideologen des Instituts nicht gefallen: er klinge zu zaristisch.

Als Gedächtnismachtzentrum sorgt sich das Institut auch um die Kontrolle der Zeit(räume). Das Institut hat viele Bemühungen unternommen, um Feier- und Gedenktage zu ändern. Der Tag des Sieges am 9. Mai wurde als sowjetisch - und damit als "fremd" - eingeordnet. Stattdessen besteht das Institut darauf, am 8. Mai den Tag der Versöhnung und der Trauer einzuführen. Diesen Tag präsentiert das Institut als einen gemeinsamen europäischen Tag, dessen Symbolik - rot-schwarzer Mohn - mit dem Farbschema der „Ukrainischen Aufständischen Armee” übereinstimmt. Auch der 8. März und der 1. Mai sind als nicht-ukrainische Feiertage deklariert worden (Radio Svoboda, 2017). Diese Feiertagsumwandlungen gehören zu den wichtigsten Zielen des Instituts (УІНП, 2018).

Die Institutsberichte für 2016 und 2017 zeigen, wie die Ereignisse, die in der Ukraine des 20. Jahrhunderts und früher geschehen sind, in zwei Kategorien unterteilt werden: „Eigenes” und „Fremdes”. Zeitweise geht „Das Unsere” aus der Kiewer Rus hervor und ist gekennzeichnet durch die Kosaken, die Ukrainische Volksrepublik, die „Ukrainische Aufständische Armee” bis hin zur heutigen, sich im Krieg befindenden Ukraine. Das Institut hat gemäß diesem Schema viele öffentliche Ausstellungen in Kiew und anderen Städten durchgeführt (sieh. z.B. Kruse, 2017). Dasselbe Schema ist in Schulbüchern und Schulveranstaltungen zu finden (УІНП, 2018).

In Bezug auf den Raum wird „Das Unsere” als Ort dargestellt, an dem mit ethnischen Ukrainern etwas Wichtiges geschah. Daher kann man auf der Webseite des Instituts Erinnerungen nicht nur aus der Ukraine, sondern auch aus Russland, Polen, Kanada oder Deutschland sehen. Gleichzeitig wurden viele Ereignisse, die in der Ukraine geschehen sind, aus dem Nationalgedächtnis gefiltert. Das betrifft fast durchgehend die Geschichte der Sowjetischen Ukraine, die nun als fremde eingeordnet wird und bezieht sich auf die Ukrainer im russischen Imperium, die sowjetische Regierung und die „Rote Armee” sowie auf russischsprachige ukrainische Schriftsteller und Künstler. Sie alle gelten nicht mehr als Teil des nationalen Gedächtnisses.

Dieselben Erinnerungsauswahlprinzipien kamen auch in der sowjetischen Historiographie zum Einsatz - allerdings mit dem Unterschied, dass nicht das ethnonationale, sondern das Klassenmerkmal angewandt wurde. 
Diese und viele andere Beispiele für vom Institut ausgeübte Funktionen belegen die ideologische Rolle dieser Staatsbehörde. Das Institut versucht, die zeitlichen und räumlichen Strukturen der ukrainischen Gesellschaft einer Reihe von ideologischen Prinzipien unterzuordnen. Die Prinzipien sind dabei stark mit einem konservativen Ethnonationalismus verknüpft, während die Institutspraktiken der bolschewistischen Praxis ähneln. Ihre Anwendung untergräbt die Möglichkeiten, eine zivile ukrainische Nation zu schaffen, die kulturelle Vielfalt des Landes zu bewahren und einen innerukrainischen Dialog zu führen.

Meine Schlussfolgerungen lauten demnach: Das heutige Ukrainische Institut für Nationales Gedächtnis ist eine Organisation zur Filterung, Kontrolle und Modifizierung der nationalen Erinnerung. Die Leitung des Institutes und die assoziierten politischen Gruppen versuchen, die ideologische Hegemonie in der Ukraine zu erlangen. Viele soziale, kulturelle, ethnische, religiöse und sprachliche Gruppen werden dadurch ignoriert und marginalisiert. Diese Aktivitäten und Versuche behindern den nationalen Dialog, die Chancen für eine liberale Demokratisierung der Ukraine und die weitere Integrierung in die Europäische Union.

\section{LITERATURVERZEICHNIS}

Acquisti Allesandro, Gross Ralph. Imagined Communities: Awareness, Information Sharing, and Priva-

cy on the Facebook. Fr./M.: Springer, 2007.

Anderson, Benedict. Imagined Communities. London: Verso Books, 1991.

Assmann Aleida, Assmann Jan, Hrsg. Verwandlungen. Archäologie der literarischen Kommunikation

IX. München: Fink, 2006.

Assmann, Jan. Das kulturelle Gedächtnis. Schrift, Erinnerung und politische Identität in frühen Hochkulturen. München: Beck, 1992.

Berek, Mathias. Kollektives Gedächtnis und die gesellschaftliche Konstruktion der Wirklichkeit. Eine

Theorie der Erinnerungskulturen. Wiesbaden: Harrassowitz, 2009.

Berger Peter, Luckmann Tomasz. The Social Construction of Reality. NY: Doubleday, 1967.

Bobrow 2017: Bobrov, Viktor, upor. Babyn Jar: pam'jat' na tli istoriï. Putivnyk dlja vchytelja: zbirnik navchal'no-metodichnih rozrobok shkil'nih zanjat' na osnovi virtual'noï vystavky ta dokumental'nogo fil'mu. Kiïv: Ukraïns'kyj centr vyvchennja istoriï Golokostu, 2017.

Geertz, Clifford. Ideology as a cultural system. NY: Free Press, 1964.

Gerhards Jürgen, Breuer Lars, Delius Anna. Kollektive Erinnerungen der europäischen Bürger im Kontext von Transnationalisierungsprozessen. Deutschland, Großbritannien, Polen und Spanien im Vergleich. Berlin: Springer, 2017.

Ghani Ashraf, Lockhart Clare. Fixing failed states: A framework for rebuilding a structured world. Oxford: Oxford University Press, 2008.

Halbwachs, Maurice. Das kollektive Gedächtnis. Frankfurt a. M.: Fischer Taschenbuch Verlag, 1985. 
Hromadske radio 2017: “Real'nyj termin za Lenina v socmerezhi i «Kapital» Marksa - podrobyci spravy'. 2017. Web. 1.12.2018. <https://hromadskeradio.org/en/news/2017/05/12/realnyy-strok-za-publikaciyu-cytat-komunistychnogo-rezhymu-u-fb-podrobyci-spravy>

Hromadske radio 2018: “Bez politichnyh gasel i provokacij: u L'vovi projshov marsh do 75-richchja diviziï «Galychyna»”. 2018. Web. 1.12.2018<https:/ / hromadskeradio.org/en/news/2018/04/28/ bez-politychnyh-gasel-i-provokaciy-u-lvovi-proyshov-marsh-do-75-richchya-dyviziyi-galychyna-foto>

Juschtschenko 2009: "Jushhenko pro Golodomor: Takogo genocydu ne znala zhodna nacija". Korrespontent. 2009. Web. 1.12.2018. <https://ua.korrespondent.net/ukraine/1022631-yushchenko-pro-golodomor-takogo-genocidu-ne-znala-zhodna-naciya>

Kasyanov 2018: Kasyanov, Georgij. Past Continuous: istorychna polityka 1980-h - 2000-h. K.: Laurus, 2018.

Kruse, Mariam. „Geschichte als Droge?“ Viadrina Goes Ukraine. Oktober 2017. Web. 1.12.2018.<https:/ / viadrinagoesukraine.wordpress.com/2017/10/11/geschichte-als-droge/>

Nora, Pierre. Erinnerungsorte Frankreichs. München: Beck, 2005.

Olick, Jeffrey. The politics of regret. On collective memory and historical responsibility. New York: Routledge, 2007.

Portnow 2014: Portnow, Andrej. “Ljustracija i instituty nacional'noj pamjati: opyt postkommunisticheskoj Evropy." 2014. Web. 1.12.2018. <http://argumentua.com/stati/lyustratsiya-i-instituty-natsionalnoi-pamyati-opyt-postkommunisticheskoi-evropy $>$

Radio Svoboda 2017: “Jaki vyhidni mozhut' z'javitisja v kraïni zamist' 8 bereznja ta 1 i 9 travnja”. 2017. Web. 1.12.2018. <https:/ / www.radiosvoboda.org/a/28267319.html>

Ricoeur, Paul. Lectures on Ideology and Utopia. NY: Columbia University Press, 1986.

Schütz Alfred, Luckmann Tomasz. Strukturen der Lebenswelt. Konstanz: UVK, 2003. 2 Bde.

Tilly, Charles. Coercion, capital, and European states, AD 990-1990. Blackwell: Oxford, 1992.

Vervassung: Konstytutcija Ukraïny. 1996/2004/2010/2014. Web. 1.12.2018. <http://iportal.rada.gov. ua/uploads/documents/27396.pdf>

Zaxid.net 2018: “Bilja LODA vidkryli vystavku pro diviziju SS «Galichina»". 2018. Web.1.12.2018. <https:/ / zaxid.net/bilya_loda_vidkrili_vistavku_pro_diviziyu_ss_galichina_n1454624>

Zik.ua 2018: “Na Donechchyni oshtrafuvaly ljudej za georgiïvs'ki strichky na 9 travnja ”. Zik.ua. 2018. Web. 1.12.2018.<zik.ua/news/2018/05/10/na_donechchyni_oshtrafuvaly_lyudey_za_georgiivski_strichky_na_9_travnya_1321295>

Kabinet Ministriv Ukraïny 2006: “Postanova vid 31 travnja 2006 r. № 764 «Pro utvorennja Ukraïns'kogo instytutu nacional'noï pam'jati»”. 2006. Web. 1.12.2018. <zakon4.rada.gov.ua/laws/ show/764-2006-п>

Kabinet Ministriv Ukraïny 2011: “Postanova vid 31 sichnja 2011 r. № 74 «Pro zatverdzhennja Polozhennja pro Ukraïns'kyj instytut nacional'noï pam'jati»". 2011. Web. 1.12.2018. <zakon4.rada.gov. ua/laws/show/74-2011-п>

Kabinet Ministriv Ukraïny 2014a: “Postanova vid 9 lipnja 2014 r. № 292 «Pytannja Ukraïns`kogo instytutu nacional'noï pam'jati»”. 2014. Web. 1.12.2018. <zakon4.rada.gov.ua/laws/show/292-2014-ח> 
Kabinet Ministriv Ukraïny 2014b: “Postanova vid 12 listopada 2014 r. № 684 «Dejaki pytannja Ukraïns'kogo instytutu nacional'noï pam'jati»”. 2014. Web. 1.12.2018. <zakon4.rada.gov.ua/laws/ show / 684-2014-ח>

UINP 2018: “Zvit Golovy Ukraïns'kogo instytutu nacional'noï pam'jati za 2017 rik”. 2018. Web. 1.12.2018. <http:/ / www.memory.gov.ua/page/zvit-za-2017-rik>

UINP 2018a: "Perelik novyh i staryh nazv naselenyh punktiv". 2018. Web. 1.12.2018. <http://www. memory.gov.ua/sites/default/files/perelik_pereymenovanih_nas_punktiv.pdf> 
